\title{
The local solvability of partial differential operator with multiple characteristics in two independent variables
}

$\mathrm{By}$

\author{
Takashi ŌKAJI
}

(Received Nov. 7, 1978)

\section{Introduction.}

Let $\Omega$ be a neighborhood of the origin in $R^{2}$ and $a(x, t)$ be an infinitely differentiable real-valued function defined in $\Omega$ of the form

$$
a(x, t)=a t^{k} a_{0}(x, t) \quad \text { for } \quad(x, t) \in \Omega .
$$

where $a$ is a non-zero real number, $k$ is a positive integer and $a_{0}(x, t)$ is an infinitely differentiable real-valued function defined in $\Omega$ with $a_{0}(0,0)=1$.

We are concerned with the operator $P$ of the form

$$
P\left(x, t, \frac{\partial}{\partial x}, \frac{\partial}{\partial t}\right)=\left(\frac{\partial}{\partial t}-i a(x, t) \frac{\partial}{\partial x}\right)^{m}+\sum_{i+j \leq m-1} b_{i, j}(x, t)\left(\frac{\partial}{\partial t}\right)^{j}\left(\frac{\partial}{\partial x}\right)^{i},
$$

where $b_{i, j}(x, t)$ are infinitely differentiable functions defined in $\Omega$.

It is well-known that for $m=1, P$ is locally solvable if and only if $k$ is even ([1], [9]). For $m \geqq 2$, there are some works [2], [4], [7] which treat more general operators. In particular, from the result of [2] it follows that for $m=2$, if $k$ is odd, then $P$ is not locally solvable at the origin. And for $m \geqq 3$, from the result of [4] it follows that when $k$ is odd, $P$ is not locally solvable at the origin if $b_{0, m-1}(0,0) \neq 0$. On the other hand in the case when $k$ is even, in [10] there is given a necessary and sufficient condition for local solvability of $P$ for $m=2$ when its coefficients depend only on variable $t$ and $b_{1,0}(x, t)=0$.

In this paper we will give a necessary and a sufficient condition for local solvability of $P$ when $m$ is two or three. In the proof of necessary part we shall use ideas of Ivrii [7] and Cardoso-Treves [2] and the proof of sufficient part relies on the result of Grusin [5].

\section{Statement of results.}

Let $\Omega_{1}$ be a neighborhood of the origin $R^{1}$ such that $\Omega_{1} \times\{0\} \subset \Omega$. For $b_{i, j} \in C^{\infty}(\Omega)$ and $x_{0} \in \Omega_{1}$ let $d_{i, j}\left(x_{0}\right)$ be a non-negative integer for which the following representation holds: In some neighborhood $\Omega^{\prime} \subset \Omega$ of $\left(x_{0}, 0\right)$ 


$$
b_{i, j}(x, t)=t^{d_{i, j}\left(x_{0}\right)} b_{i, j}^{0}(x, t) \quad \text { for } \quad(x, t) \in \Omega^{\prime}, \quad b_{i, j} \in C^{\infty}\left(\Omega^{\prime}\right)
$$

where $b_{i, j}^{0}(x, 0)$ is not identically zero in every small neighborhood of $\left(x_{0}, 0\right)$. If such an integer does not exist, we define $d_{i, j}\left(x_{0}\right)$ to be $+\infty$.

Now we state our theorems.

Theorem 2.1-0. Let $P$ be the operator of (1.2), and $m$ be less than or equal to three. Then $P$ is locally solvable at the origin if $k$ is even and $d_{i, j}(0) \geqq i+j(1+k)$ $-m$ for every $i, j$ such that $i+j \leqq m-1$.

Remark 2.3. The condition of the theorem $2.1-0$ is invariant if we replace $P$ by ${ }^{t} P$.

In this paper we will give the proof in only the case when $m=3$ because the proof of the case $m=2$ is essentially the same as $m=3$ and is easier than $m=3$. Henceforth we assume $m=3$.

Concerning the necessity of the condition in th $2.1-0$, we have the following theorem.

Theorem 2.1-1. ${ }^{t} P$ is not locally solvable at the origin if there exists $(i, j)$ such that $d_{i, j}(0)<i+j(1+k)-3$, and the condition A), B) or C) holds, where A), $\mathrm{B}), \mathrm{C})$ is given in $\S 4$.

Theorem 2.1-2. $P$ is not locally solvable at the origin if $k$ is odd and for every $(i, j)$ such that $i+j \leqq 2, d_{i, j}(0) \geqq i+j(1+k)-3$ holds.

Remark 2.4. In theorem $2.1-1$ and $2.1-2$, if $k$ is odd, then we can weaken the hypothesis on $a_{0}(x, t)$. Namely, in place of $a_{0}(0,0)=1$, it is enough to assume that $a_{0}(x, 0)$ is not identically zero in every small neighborhood of the origin.

In section 3, 4, 5, we will prove the non local-solvable result, i. e. theorem $2.1-1,2.1-2$. In the last section we will prove theorem $2.1-0$.

\section{Basic inequality.}

We will prove the non-solvability of $P$ by contradiction. The method relies on the following lemma.

Lemma 3.1. (See [6].) Svppose that ${ }^{t} P$ is locally solvable at the origin. Then there are a neighborhood $V$ of the origin, and constants $C, M$ such that the inequality

$$
\left|\iint f(x, t) v(x, t) d x d t\right| \leqq C|f(x, t)|_{M}|P v(x, t)|_{M}
$$

is valid for all $f, v \in C_{0}^{\infty}(V)$. In (3.1), we denote $\sup _{i+j \leq M}\left|\left(\frac{\partial}{\partial t}\right)^{i}\left(\frac{\partial}{\partial x}\right)^{j} u(x, t)\right| b y$ $|u(x, t)|_{M}$. 
Now as in [2], we are going to perform an analytic approximation of coefficients of $P$. Let $J$ be a positive integer. We replace each coefficient of $P$ by its finite Taylor expansion of order $J+M$ about $(0,0)$. For simplicity of notation we will continue to denote the approximated operator by $P$. Then the inequality (3.1) becomes

$$
\left|\iint f v d x d t\right| \leqq C|f|_{M}\left\{|P v|_{M}+C^{\prime} \sup _{V}(|x|+|t|)^{J}|v|_{M+3}\right\} .
$$

Here we make use of asymptotic change of variables. (c. f. see [7].) Let us introduce new variables $(y, s)$ as follows:

$$
s=\rho^{\lambda} t, \quad y=\rho^{\mu} x,
$$

where $\lambda$ and $\mu$ are the positive real number which are suitably chosen later in various ways, and $\rho$ is a large parameter. Then in new variables $(y, s)$ we have

Lemma 3.2. Suppose that ${ }^{~} P$ is locally solvable at the origin. Then for every open set in $\boldsymbol{R}^{2}$ whose closure is compact, there exist constant $C, M, M^{\prime}$ and $\rho_{0}$ such that the inequality

$$
\left|\iint f v d y d s\right| \leqq C \rho^{M \prime}|f|_{M}\left\{\left|P_{\rho} v\right|_{M}+C \sup _{U}\left(\left|\rho^{-\lambda} s\right|+\left|\rho^{-\mu} y\right|\right)^{J}|v|_{M+3}\right\}
$$

is valid for $f, v \in C_{0}^{\infty}(U)$ and $\rho \geqq \rho_{0}(U)$, where $P_{\rho}$ is obtained from the analytic approximated operator $P$ after change of variables (3.3):

$$
\begin{aligned}
& \rho^{-3 \lambda} P_{\rho}(y, s)=\left(\frac{\partial}{\partial s}-\rho^{-n_{0} i a s^{k}} a_{0}\left(\rho^{-\mu} y, \rho^{-\lambda} s\right) \frac{\partial}{\partial y}\right)^{3}
\end{aligned}
$$

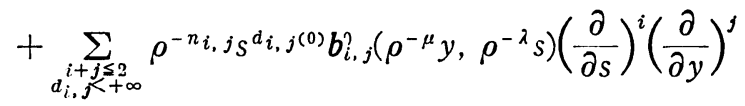

$$
\begin{aligned}
& n_{0}=(1+k) \lambda-\mu, \quad n_{i, j}=\left(d_{i, j}(0)+3-i\right) \lambda-j \mu .
\end{aligned}
$$

We note that in (3.5) $a_{0}(y, s)$ and $b_{i, j}^{0}(y, s)$ are polynomials in $y, s$.

\section{Proof of theorem 2.1-1.}

In this section, for simplicity of notation, we denote $d_{i, j}(0)$ by $d_{i, j}$.

Now we will construct $f$ and $v$ for which (3.4) does not hold. But its construction depends on what lower order terms have the strongest influence.

Lemma 4.1. If there exists $(i, j)$ such that $d_{i, j}<i+j(1+k)+3$, then at least one of the following conditions i)-iii) holds.

i) $d_{1,1}<k-1, d_{1,1}+k \leqq d_{0,2}$ and $2 d_{1,1} \leqq d_{0,1}+k$

ii) $d_{0,2}<2 k-1, d_{0,2}<d_{1,1}+k$ and $2 d_{0,2} \leqq d_{0,1}+3 k$

iii) $d_{0,1}<k-2, d_{0,1}+k<2 d_{1,1}$ and $d_{0,1}+3 k<2 d_{0,2}$

Proof. For simplicity of notation, we write $d_{1}=d_{1,1}, d_{2}=d_{0,2}$, and $d_{3}=d_{0,1}$. Lemma is easily shown because we can define a totally order relation $\prec$ as 
follows :

$$
\begin{array}{ccc}
d_{i} \prec d_{i} & i=1,2,3 . \\
d_{1} \prec d_{2} & \text { if } & d_{1}+k \leqq d_{2} . \\
{[\succ]} & & {[\geqq]} \\
d_{2} \prec d_{3} & \text { if } & 2 d_{2} \leqq 3 k+d_{3} . \\
{[\succ]} & & {[\geqq]} \\
d_{1} \prec d_{3} & \text { if } & 2 d_{1} \leqq d_{3}+k . \\
{[\succ]} & & {[\geqq]}
\end{array}
$$

From this lemma, it is sufficient to prove that for each case of i)-iii) ${ }^{t} P$ is not locally solvable at the origin, if some additinal condition holds.

First, we consider the case i). In this case the term $\frac{\partial}{\partial s} \frac{\partial}{\partial y}$ has the important influence. So in view of (3.5) we take $\lambda, \mu$ of (3.3) in such a way that $n_{0}=1 / 2, n_{1,1}=0$ :

$$
\begin{aligned}
& \lambda=1 / 2\left(k-1-d_{1,1}\right) \\
& \mu=\left(d_{1,1}+2\right) / 2\left(k-1-d_{1,1}\right) .
\end{aligned}
$$

Then we note that the number $\lambda, \mu$ are positive by hypothesis. Let $P_{\rho}^{\prime}=\rho^{-3 \lambda} P_{\rho}$. We are going to construct the approximate null solution $u_{\rho}^{N}$ of $P_{\rho}^{\prime} \mu=0$ of the form.

$$
u_{\rho}^{N}(y, s)=e^{i w_{\rho}^{N}(y, s)}, \quad \text { where } \quad w_{\rho}^{N}(y, s)=\rho z_{0} y+\sum_{j=1}^{N} \rho^{-i / 2} h_{\rho}^{j}(y, s) .
$$

In the above, $z_{0}$ which is a nonzero real number and $h_{\rho}^{j}(y, s) \in C^{\infty}(U)$ which are bounded as $\rho \rightarrow+\infty$ are determined later, and $U$ is also determined later.

For simplicity of notation, we define $c_{j}(j=1,2,3)$ as follows:

$$
\begin{aligned}
& c_{1}=b_{1,1}^{0}(0,0) \\
& c_{2}=\left\{\begin{array}{lll}
b_{0,2}^{0}(0,0) & \text { if } & d_{0,2}=d_{1,1}+k, \\
0 & \text { if } & d_{0,2}>d_{1,1}+k .
\end{array}\right. \\
& c_{3}=\left\{\begin{array}{lll}
b_{0,1}^{0}(0,0) & \text { if } & d_{0,1}=2 d_{1,1}-k, \\
0 & \text { if } & d_{0,1}>2 d_{1,1}-k .
\end{array}\right.
\end{aligned}
$$

By calculation, we have

$$
e^{-i w_{\rho}^{N}} P_{\rho}^{\prime} u_{\rho}^{N}=\sum_{j=1}^{N+1} \rho^{(3 / 2)-j} A_{\rho}^{j}\left(h_{\rho}^{-1}, \cdots, h_{\rho}^{-1+j}\right)+\rho^{(N-1) / 2} B_{\rho}\left(h_{\rho}^{-1}, \cdots, h_{\rho}^{N}\right)
$$

where $A_{\rho}^{j}$ and $B_{\rho}$ are nonlinear differential operators acting on $h_{\rho}^{-1}, \cdots h_{\rho}^{-1+j}$ and $h_{\rho}^{-1}, \cdots, h_{\rho}^{N}$, respectively, and $A_{\rho}^{j}\left(h_{\rho}^{-1}, \cdots, h_{\rho}^{-1+j}\right)$ and $B_{\rho}\left(h_{\rho}^{-1}, \cdots, h_{\rho}^{N}\right)$ are bounded as $\rho \rightarrow+\infty$. More precisely, 


$$
\begin{aligned}
A_{\rho}^{0}\left(h_{\rho}^{-1}\right)= & \left\{i\left(\frac{\partial}{\partial s} h_{\rho}^{-1}\right)+z_{0} a s^{k} a_{0}\left(\rho^{-\mu} y, \rho^{-\lambda} s\right)\right\}^{3} \\
+ & \left(-c_{1} s^{d_{1,1}} z_{0}+\rho^{-r_{1}} H_{\rho}(y, s)\right) \frac{\partial}{\partial s} h_{\rho}^{-1} \\
- & c_{2} s^{d_{1,1}+k} z_{0}^{2}+i c_{3} s^{2 d_{1,1}-k} z_{0}+\rho^{-r_{2}} G_{\rho}^{0}(y, s) \\
A_{\rho}^{j}\left(h_{\rho}^{-1}, \cdots, h_{\rho}^{-1+j}\right)= & {\left[3 i\left\{i\left(\frac{\partial}{\partial s} h^{-1}\right)+z_{0} a s^{k} a_{0}\left(\rho^{-\mu} y, \rho^{-\lambda} s\right)\right\}^{2}\right.} \\
& \left.+\left(-c_{1} z^{d_{1,1}} z_{0}+\rho^{-r_{1}} H_{\rho}(y, s)\right)\right] \frac{\partial}{\partial s} h_{\rho}^{-1+j} \\
& +G_{\rho}^{j}\left(y, s, h_{\rho}^{-1}, \cdots, h_{\rho}^{-2+j}\right),
\end{aligned}
$$

where $r_{1}$ and $r_{2}$ are some positive numbersm and $H_{\rho}(y, s)$ and $G_{\rho}^{j}\left(y, s, h_{\rho}^{-1}, \cdots\right.$, $h_{\rho}^{-2+j}$ ) are smooth in $U$ in which $h_{\rho}^{-1}, \cdots, h_{\rho}^{-2+j}$ are smooth and are uniformly bounded in $U$ as $\rho \rightarrow+\infty$.

We want to determine $h_{\rho}^{-1}, \cdots, h_{\rho}^{N}$ in such a way that $e^{-i w_{\rho}^{N}} P_{\rho}^{\prime} u=0\left(\rho^{-(N-1) / 2}\right)$. Hence we are going to determine $h_{\rho}^{j}$ such that

$$
\begin{aligned}
& A_{\rho}^{0}\left(h_{\rho}^{-1}\right)=0\left(\rho^{-(N-1) / 2}\right) \\
& A_{\rho}^{j}\left(h_{\rho}^{-1}, \cdots, h_{\rho}^{-1+j}\right)=0
\end{aligned}
$$

First we consider the equation (4.6). Let us define $X_{\rho}(y, s)$ as follows.

$$
X_{\rho}(y, s)=i\left(\frac{\partial}{\partial s} h_{\rho}^{-1}\right)+a s^{k} a_{0}\left(\rho^{-\mu} y, \rho^{-\lambda} s\right) z_{0} .
$$

Then for some $r>0$, (4.4) becomes

$$
X_{\rho}^{3}+\left\{p(s)+\rho^{-r} \tilde{H}_{\rho}(y, s)\right\} X_{\rho}+q(s)+\rho^{-r} \tilde{G}_{\rho}(y, s)
$$

where $p(s)=i c_{1} s^{d_{1,1}} z_{0}$, and $q(s)=-\left(i c_{1} a+c_{2}\right) s^{d_{1,1}+k} z_{0}^{2}+i c_{3} s^{2 d_{1,1}{ }^{k}} z_{0}$, and $\widetilde{H}_{\rho}(y, s)$. $\tilde{G}_{\rho}(y, s)$ are smooth and bounded as $\rho \rightarrow+\infty$. Then we are going to determine $X_{\rho}$ such that (4.6) holds having the form

$$
X_{\rho}(y, s)=X^{0}(s)+\sum_{j=1}^{N_{0}} \rho^{-r i} X_{\rho}^{j}(y, s),
$$

where $N_{0}$ is a positive integer such that $r N_{0}>1 / 2(N-1)$. Substitute (4.10) into (4.9). Then we get

$$
\begin{gathered}
\left(X^{0}\right)^{3}+p(s) X^{0}+q(s)+\sum_{j=1}^{N_{0}} \rho^{-r j}\left\{3\left(X^{0}\right)^{2} X^{j}+E_{\rho}^{j}\left(X^{0}, \cdots, X_{\rho}^{j-1}\right)\right. \\
\left.+\rho^{-\left(N_{0}+1\right)} r E_{\rho}^{N_{0}+1}\left(X^{0}, \cdots, X_{\rho}^{N_{0}}\right)\right\}
\end{gathered}
$$

where $E_{\rho}^{j}\left(X_{0}, \cdots, X_{j-1}\right)$ are bounded as $\rho \rightarrow+\infty$. Therefore if $X^{0}$ and $X_{\rho}^{j}$ are the roots of the equations

$$
\begin{aligned}
& \left(X^{0}\right)^{3}+p(s) X^{0}+q(s)=0 \\
& 3\left(X^{0}\right)^{2} X_{\rho}^{j}+E_{\rho}^{j}\left(X^{0}, \cdots, X_{\rho}^{j-1}\right)=0,
\end{aligned}
$$


then (4.6) holds.

Now let us analyze the equation (4.12) for $s>0$. We assume (C.1);

(C.1) There exists a non-zero real number $z_{0}$ which satisfies the following three conditions.

i) Either $c_{3} \neq 0$ or $c_{3}=0$ and $i c_{1} z_{0} \notin \boldsymbol{R}_{+}$.

ii) $\left(a z_{0}\right)>0$.

iii) the discriminant $D$ of the equation (4.12) is not zero for every $s>0$. where by calculation we have

$$
\begin{aligned}
D & =-27\left\{q^{2}+4(p / 3)^{3}\right\} \\
& =-27\left\{C_{1}\left(z_{0} s^{2 k-d_{1,1}}\right)^{2}+C_{2}\left(z_{0} s^{2 k-d_{1,1}}\right)+C_{3}\right\} z_{0}^{2} s^{4 d_{1,1-2 k}}
\end{aligned}
$$

where $C_{1}=\left(-i c_{1} a-c_{2}\right)^{2}, C_{2}=2 i c_{3}\left(-i c_{1} a-c_{2}\right)+4\left(i c_{1} / 3\right)^{3}$, and $C_{3}=\left(i c_{3}\right)^{2}$. We remark that if $\left|c_{1}\right|+\left|c_{2}\right|+\left|c_{3}\right| \neq 0$, then $C_{1}, C_{2}$ and $C_{3}$ are not simultaneously zero.

Lemma 4.3. There exists a simple root $X^{0}$ of the equation (4.12) such that

$$
\begin{array}{ll}
\operatorname{Re} X^{0}(s)=c s^{d}(1+o(s)) & \text { for sufficiently small } s>0 \\
\left|X^{0}(s)\right| \leqq c^{\prime} s^{d \prime} & \text { for sufficiently large } s>0,
\end{array}
$$

where $c$ and $c^{\prime}$ are positive constants and $0 \leqq d, d^{\prime}<k$.

Proof. First we consider $X(s)$ for small $s>0$. In the case $c_{8}=0$, we set $X(s)=s^{(1 / 2) d_{1,1}} Y_{1}(s)$. Then $Y_{1}(s)$ satisfies the equation

$$
\left(Y_{1}(s)\right)^{3}+i c_{1} z_{0} Y_{1}(s)+\left(-i c_{1} a-c_{2}\right) z_{0}^{2} s^{k-(1 / 2) d_{1,1}}=0
$$

From this, we can choose $Y_{1}(s)$ such that $\operatorname{Re} Y_{1}(0)$ is positive because $i c_{1} z_{0}$ is not in $\boldsymbol{R}_{+}$. In the case $c_{3} \neq 0$, we set $X(s)=s^{(1 / 3)\left(2 d_{1,1}-k\right)} Y_{2}(s)$. Then

$$
\left(Y_{2}(s)\right)^{3}+i c_{1} z_{0} s^{k-d_{1,1}} Y_{2}(s)+i c_{3} z_{0}+\left(-i c_{1} a-c_{2}\right) s^{2 k-d_{1,1}} z_{0}^{2}=0
$$

In this case too, we can choose $Y_{2}(s)$ such that $\operatorname{Re} Y_{2}(0)$ is positive.

On the other hand, for large $s>0$, we set $X(s)=s^{(1 / 3)\left(d_{1,1}+k\right)} Z(s)$. Then

$$
(Z(s))^{3}+i c_{1} z_{0} s^{-k} Z(s)+\left(i c_{1} a-c_{2}\right) z_{0}+i c_{3} s^{d_{1,1-2 k}} z_{0}=0 .
$$

Since $d_{1.1}-2 k$ is negative, all the roots of this equation are bounded as $s \rightarrow+\infty$. These considerations and (C.1) give the proof of lemma.

Let $X^{0}(s)$ be the simple root of (4.12) which satisfies (4.14) (4.15). Let us consider the following continuous function:

$$
I_{1}(s)=\frac{a}{k+1} \operatorname{Re} z_{0} s^{k+1}-\operatorname{Re}\left\{\int_{0}^{s} X^{0}(t) d t\right\} .
$$


Then from (4.14), (4.15), it follows that $I_{1}(s)$ has a negative minimum value $m_{0}$ at $s_{0}>0$ in $s>0$. Let $U_{1}$ be a neighbourhood of $s_{0}$ contained in $\{s>0\}$ in which $I_{1}(s)$ is negative and $I_{1}(s)-m_{0}>0$ except for $s=s_{0}$. We note that the existence of such a neighborhood is insured by the analyticity of $I_{1}(s)$ for $s>0$. Then $X^{0}(s)$ does not vanish in $U_{1}$. Therefore by (4.13) we can determine $X_{\rho}^{j}(y, s)$ which is smooth in $R^{1} \times U_{1}\left(j=1, \cdots, N_{0}\right)$.

Now we determine $h_{\rho}^{-1}$ which satisfies the equation

$$
\frac{\partial}{\partial s} h^{-1}=i a s^{k} a_{0}\left(\rho^{-\mu} y, \rho^{-\lambda} s\right) z_{0}-i X_{\rho}(y, s) .
$$

Since $a_{0}(y, s)$ is a polynomial in $y, s$, there exists a positive number $r^{\prime}$ for which (4.17) can be written as follows:

$$
\frac{\partial}{\partial s} h^{-1}=i a s^{k} z_{0}-i X^{0}(s)+\rho^{-r^{\prime}} K_{\rho}(y, s),
$$

where $K_{\rho}$ is uniformly bounded in $U^{\prime} \times U_{1}$ as $\rho \rightarrow+\infty$. $\left(\bar{U}^{\prime}\right.$ is any compact set in $\boldsymbol{R}^{1}$ ). So that $h_{\rho}^{-}$are determined as follows :

$$
h_{\rho}^{-1}(y, s)=\frac{i a}{k+1} s^{k+1} z_{0}-i \int_{0}^{s} X^{0}(t) d t+\rho^{-r \prime} \int_{s_{0}}^{s} K_{\rho}(y, t) d t+i y^{2} .
$$

Let $U_{2}$ be a sufficiently small neighborhood of $y=0$, and we set $U=U_{2} \times U_{1}$. Then $h_{\rho}^{-1}(y, s)$ is smooth in $U$.

Now we are going to determine $h_{\rho}^{j}(j=0,1, \cdots, N)$. Since $X^{0}$ is a simple root, there exists $\rho_{0}>0$ such that for $\rho>\rho_{0}$, the coefficient of $h_{\rho}^{-1+j}$ in equation (4.5) does not vanish in $U$. So we can determine $h_{\rho}^{-1+j}$ inductively by the equation (4.5). Then $h_{\rho}^{-1+j}(y, s)$ is smooth in $U$.

Now as in [2], let us consider the following function $I(y, s)$.

$$
\begin{aligned}
I(y, s) & =\rho\left(\operatorname{Im} z_{0}\right) y+\rho^{(1 / 2)} \operatorname{Im} h_{\rho}^{-1}(y, s) \\
& =\rho^{(1 / 2)}\left\{I_{1}(s)^{\prime}+y^{2}+\rho_{\rho}^{-r^{\prime}} \operatorname{Im}\left[\int_{s_{0}}^{s} K_{\rho}(y, t) d t\right]\right\} .
\end{aligned}
$$

Then $I(y, s)$ has a minimum value $m(\rho)$ at $\left(y_{0}(\rho), s_{0}(\rho)\right)$ in $U$. Moreover

$$
\left(y_{0}(\rho), s_{0}(\rho)\right) \text { converges to }\left(0, s_{0}\right) \text { as } \rho \longrightarrow+\infty \text {, }
$$

and for sufficiently large $\rho$, we have

$$
I(y, s)-m(\rho)>C \rho^{(1 / 2)} \quad \text { for } \quad(y, s) \in\left\{(y, s) \in U ;|y|+\left|s-s_{0}\right|>\varepsilon\right\},
$$

where $C, \varepsilon$ are some positive constants.

We define $v, f$ as follows:

$$
\begin{aligned}
& v(y, s)=g(y, s) u_{\rho}^{N}(y, s) \\
& f(y, s)=F\left(\rho y, \rho\left(s-s_{0}(\rho)\right)\right),
\end{aligned}
$$

where $g(y, s)$ is a smooth function defined in $\boldsymbol{R}^{2}$ with compact support contained in $U$ and equals to one in a subneighborhood of $\left(0, s_{0}\right)$, and $F(y, s)$ belongs to 
$C_{0}^{\infty}\left(\boldsymbol{R}^{2}\right)$ and satisfies $\iint e^{i z_{0} y} F(y, s) d y d s=1$.

Then in the usual way, by (4.3), (4.6), (4.7), (4.21), (4.22) we can show that for $v, f$ of (4.23), (4.24),

$$
\text { the right hand side of }(3.4) \leqq 0\left(\rho^{-J \cdot(N, J)}\right) e^{-m(\rho)} \text {, }
$$

where $J^{\prime}(N, J)$ tends to $+\infty$ as $N, J \rightarrow+\infty$, and

the left hand side of $(3.4) \geqq C \rho^{-2} e^{-m(\rho)}$,

where the constant $C$ is not zero. (c.f. see [2]). Therefore if we choose $J$ and $N$ such that $J^{\prime}(J, N)>2$, the inequality (3.4) never holds.

In other cases of lemma 4.1, the proofs are essentially the same as the case i). Therefore we will outline them and the detail is omitted.

In the case $\mathrm{ii}$ ), we take $\lambda, \mu$ such that $n_{0}=1 / 3, n_{0,2}=0$ :

$$
\begin{aligned}
& \lambda=\frac{2}{3\left(2 k-1-d_{0,2}\right)} \\
& \mu=\frac{d_{0,2}+3}{3\left(2 k-1-d_{0,2}\right)},
\end{aligned}
$$

and we will construct the approximate null solution $u_{\rho}^{N}$ of $P_{\rho}^{\prime} u=0$ of the form

$$
u_{\rho}^{N}(y, s)=e^{i w_{\rho}^{N}(y, s)}, \quad \text { where } \quad w_{\rho}^{N}(y, s)=\rho z_{0} y+\sum_{j=-2}^{N} \rho^{-(j / 3)} h_{\mu}^{j}(y, s) .
$$

In the case $\mathrm{iii}$ ), we take $\lambda, \mu$ such that $n_{0}=2 / 3, n_{0,1}=0$ :

$$
\begin{aligned}
& \lambda=\frac{2}{3\left(k-2-d_{0,1}\right)} \\
& \mu=\frac{2\left(d_{0,1}+3\right)}{3\left(k-2-d_{0,1}\right)},
\end{aligned}
$$

and we will construct the approximate null solution $u_{\rho}^{N}$ of $P_{\rho}^{\prime} u=0$ of the form

$$
u_{\rho}^{N}(y, s)=e^{i w_{\rho}^{N}}(y, s), \quad \text { where } \quad w_{\rho}^{N}(y, s)=\rho z_{0} y+\sum_{j=-1}^{N} \rho^{-(j / 3)} h_{\rho}^{j}(y, s) .
$$

Summing up the above arguements, we have,

Theorem 2.1-1. ${ }^{t} P$ is not locally solvable at the origin if the following condition A), B) or C) holds.

A) i) of lemma 4.1 and (C.1) hold.

B) ii) of lemma 4.1 holds and either $b_{0,2}^{0}(0,0) \neq 0$ and $i c_{3} / b_{0,2}^{0}(0,0) \notin a \boldsymbol{R}_{+}$or $b_{0,2}^{0}(0,0)=0$ and $c_{3}^{\prime} \neq 0$.

C) iii) of lemma 4.1 holds and $b_{0,1}^{0}(0,0) \neq 0$.

where $c_{3}^{\prime}=0$ if $2 d_{0,2}<d_{0,1}+3 k$ and $c_{3}^{\prime}=b_{0,1}^{0}(0,0)$ if $2 d_{0,2}=d_{0,1}+3 k$.

\section{Proof of theorem 2.1-2.}

In this section we will prove that ${ }^{t} P$ is not locally solvable at the origin if $P$ satisfies the hypothesis of theorem 2.1-2. Then in view of remark 2.3 , this 
proves theorem 2.1-2.

Suppose ${ }^{t} P$ is locally solvable at the origin. Then by lemma 3.2, there exist $C, M, M^{\prime}$ and $\rho_{0}$ such that the inequality (3.4) holds for $f, v \in C_{0}^{\infty}(U)$ and $\rho \geqq \rho_{0}$.

In this case we set

$$
\mu=(1+k) \lambda, \quad \text { where } \lambda \text { is a large positive number determined later. }
$$

Then by the hypothesis of theorem $2.1-2, P_{\rho}^{\prime}$ can be written as follows:

$$
\begin{aligned}
P_{\rho}^{\prime}= & \left(-\frac{\partial}{\partial s}-i a s^{k} \frac{\partial}{\partial y}\right)^{3}+\sum_{i+j \leq 2} c_{i, j} s^{i+j(1+k)-3}\left(\frac{\partial}{\partial s}\right)^{i}\left(\frac{\partial}{\partial y}\right)^{j} \\
& +\rho^{-r(\lambda)} Q_{\rho}\left(y, s, \frac{\partial}{\partial y}, \frac{\partial}{\partial s}\right),
\end{aligned}
$$

where $c_{i, j}$ are constants, $r(\lambda)$ tends to $+\infty$ as $\lambda \rightarrow+\infty$ and $Q_{\rho}$ is a differential operator of order 3 with analytic coefficients which depend on $\rho$ but are uniformly bounded in every compact set as $\rho \rightarrow+\infty$.

We take $\lambda$ such that

$$
r(\lambda)-1>3 /(k+1) .
$$

Here we remark that without loss of generality we may assume a is positive. Now we are going to construct the approximate null solution $u_{\rho}^{N}$ of the equation $P_{\rho}^{\prime} u=0$. We require that $u_{\rho}^{N}$ has the form:

$$
\begin{aligned}
& u_{\rho}^{N}(y, s)=e^{i \rho w(y, s)} \sum_{j=0}^{N} \rho^{-j} \varphi_{\rho}^{j}(y, s), \\
& w(y, s)=\frac{i a}{k+1} s^{k+1}+y+i\left(\frac{i a}{k+1} s^{k+1}+y\right)^{2} .
\end{aligned}
$$

Then it is easily seen that

$$
\begin{aligned}
P_{\rho}^{\prime} u_{\rho}^{N}= & \sum_{j=1}^{N} \rho^{-j}\left[\left(\frac{\partial}{\partial s}-i a s^{k} \frac{\partial}{\partial y}\right)^{3} \varphi_{\rho}^{j}+s^{k-1} A_{1}\left(y, s, \frac{\partial}{\partial y}, \rho\right) \frac{\partial}{\partial s} \varphi_{\rho}^{j}\right. \\
& +\left\{s^{2 k-1} A_{2}\left(y, s, \frac{\partial}{\partial y}, \rho\right)+s^{k-2} A_{3}\left(y, s, \frac{\partial}{\partial y}, \rho\right)\right\} \varphi_{\rho}^{j} \\
& \left.+\rho^{-r(\lambda)+1} C_{\rho}^{j}\left(y, s, \varphi_{\rho}^{j-1}\right)\right] e^{i \rho w}+\rho^{-(r(\lambda)+N)} G_{\rho}^{j+1}\left(y, s, \varphi_{\rho}^{N}\right) e^{i \rho w},
\end{aligned}
$$

where $A_{n}(n=1,2,3)$ has the form

$$
\begin{aligned}
& A_{n}=c_{n}^{1}(y, s) \rho+c_{n}^{2}(y, s) \frac{\partial}{\partial y} \quad \text { for } n=1,3, \\
& A_{2}=c_{2}^{1}(y, s) \rho^{2}+c_{2}^{2}(y, s) \rho \frac{\partial}{\partial y}+c_{2}^{3}(y, s)\left(\frac{\partial}{\partial} \frac{\partial}{y}\right)^{2}
\end{aligned}
$$

and $G_{\rho}^{j}\left(y, s, \varphi_{\rho}^{j-1}\right)=e^{-i \rho w} Q\left[e^{i \rho w} \varphi_{\rho}^{j-1}\right]$. In (5.6), $c_{j}^{i}(y, s)$ are polynomials in $y, s$.

We show now how the analytic function $\varphi_{\rho}^{j}$ is chosen. Let $\varphi_{\rho}^{j}(y, s)$ be a solution of the following equation 


$$
\begin{gathered}
\left(\frac{\partial}{\partial s}-i a s^{k} \frac{\partial}{\partial y}\right)^{3} \varphi_{\rho}^{j}+s^{k-1} A_{1} \frac{\partial}{\partial s} \varphi_{\rho}^{j}+\left(s^{2 k-1} A_{2}+s^{k-2} A_{3}\right) \varphi_{\rho}^{j} \\
+\rho^{-r(\lambda)+1} G_{\rho}^{j}\left(y, s, \varphi_{\rho}^{j-1}\right)=0 .
\end{gathered}
$$

Moreover we require that $\varphi_{\rho}^{0}(0,0)=1, \varphi_{\rho}^{j}(0,0)=0$ for $j>0$.

Here we consider $(y, s)$ as complex variables, and we perform a holomorphic change of variables from $(y, s)$ into $(z, s)$ as follows:

$$
z=\frac{i a}{k+1} s^{k+1}+y, \quad s=s .
$$

Then in new variables $(z, s)$, (5.7) becomes

$$
\left(\frac{\partial}{\partial s}\right)^{3} \varphi_{\rho}^{j}+s^{k-1} B_{1} \frac{\partial}{\partial s} \varphi_{\rho}^{j}+\left(s^{2 k-1} B_{2}+s^{k-2} B_{3}\right) \varphi_{\rho}^{j}+\rho^{-r(\lambda)+1} G_{\rho}^{j^{*}}\left(z, s, \varphi_{\rho}^{j-1}\right)=0
$$

where for simplicity of notation we denote the transformed $\varphi_{\rho}^{j^{*}}$ by $\varphi_{\rho}^{j}$ and $B_{n}\left(z, s, \frac{\partial}{\partial z}, \rho\right)$ has the similar form to $A_{n}$ :

$$
\begin{aligned}
& B_{n}=b_{n}^{1}(z, s) \rho+b_{n}^{2}(z, s) \frac{\partial}{\partial z} \quad \text { for } \quad n=1,3, \\
& B_{2}=b_{2}^{1}(z, s) \rho^{2}+b_{2}^{2}(z, s) \rho \frac{\partial}{\partial z}+b_{2}^{3}(z, s)\left(\frac{\partial}{\partial z}\right)^{2} .
\end{aligned}
$$

In order to fulfill the requirement for $\varphi_{\rho}^{j}$, we require

$$
\varphi_{\rho}^{0}(z, 0)=1, \frac{\partial}{\partial s} \varphi_{\rho}^{0}(z, 0)=\left(\frac{\partial}{\partial s}\right)^{2} \varphi_{\rho}^{0}(z, 0)=0,\left(\frac{\partial}{\partial s}\right)^{i} \varphi_{\rho}^{j}(z, 0)=0,
$$

Then we have

$$
(i=0,1,2) \text {. }
$$

Proposition 5.1. Let $\varphi^{j}(z, s)$ be a solution of equation $(5.9)_{j}$ with initial data (5.11). Then for a sufficiently small neighborhood of the origin $V \subset C^{2}$, and for every $\varepsilon>0$, there exists a constant $C_{i, j^{\prime}, \varepsilon}$ such that the following estimate holds: for every $i, i^{\prime}$,

$$
\left|\left(\frac{\partial}{\partial s}\right)^{i}\left(\frac{\partial}{\partial z}\right)^{i \cdot} \varphi^{j}(z, s)\right| \leqq C_{i, i^{\prime}, \varepsilon} \rho^{i /(k+1)} e^{\varepsilon \rho|s|^{k+1}} \quad \text { for } \quad(z, s) \in V .
$$

Proof. First we consider $\varphi^{0}(z, s)$. We define $\varphi_{n}^{0}(n=0,1,2, \cdots)$ as follows:

$$
\left\{\begin{array}{l}
\varphi_{0}^{0}=1 \\
\varphi_{n}^{0}=1+\int_{0}^{s} \frac{1}{2}(s-t)^{2}\left\{t^{k-1} B_{1} \frac{\partial}{\partial t} \varphi_{n-1}^{0}+\left(t^{2 k-1} B_{2}+t^{k-2} B_{3}\right) \varphi_{n-1}^{0}\right\} d t .
\end{array}\right.
$$

Here we note that $\varphi_{n}^{0}(z, s)$ is analytic. Let $\phi_{n}^{0}=\varphi_{n}^{0}-\varphi_{n-1}^{n}$. Then $\psi_{n}^{0}$ satisfies the following equations.

$$
\left\{\begin{array}{l}
\phi_{0}^{0}=1 \\
\phi_{n}^{0}=\int_{0}^{s} \frac{1}{2}(s-t)^{2}\left\{t^{k-1} B_{1} \frac{\partial}{\partial t} \psi_{n-1}^{0}+\left(t^{2 k-1} B_{2}+t^{k-2} B_{3}\right) \psi_{n-1}^{0}\right\} d t .
\end{array}\right.
$$


Let $V_{R}$ be a neighborhood $\{x \in C ;|x|<R\}$, and we denote $V_{R_{1}} \times V_{R_{2}}$ by $V_{R_{1}, R_{2}}$. Let $R$ be a positive number such that for some positive constant $c$, the following inequality hold:

$$
\left\{\begin{array}{l}
\left|b_{n}^{i}(z, s)\right|<c \quad(n=1,3 \text { and } i=1,2) \\
\left|b_{2}^{i}(z, s)\right|<c^{2} \quad(i=1,2,3) \quad \text { for } \quad(z, s) \in V_{4 R, 4 R} . \\
\frac{1}{R} 3 c|s|^{k+1}<1, \frac{2}{R^{2}} 3 c|s|^{k+1}<1
\end{array}\right.
$$

Lemma 5.2. (Cauchy's inequality) Suppose that $f(z, s)$ is analytic in $\{(z, s) \in$ $\left.C^{2} ;\left|z-z^{\prime}\right|<r_{1},\left|s-s^{\prime}\right|<r_{2}\right\}$. Then the following inequality holds.

$$
\left|\left(\frac{\partial}{\partial s}\right)^{i}\left(\frac{\partial}{\partial z}\right)^{i^{\prime}} f\left(z^{\prime}, s^{\prime}\right)\right| \leqq \frac{i ! i^{\prime} !}{r_{1}^{i^{\prime}} r_{2}^{i}} \sup _{\substack{\left|z-z^{\prime}\right|<r_{1} \\\left|s-s^{\prime}\right|<r_{2}}}|f(z, s)|
$$

Then from this lemma, for an analytic function $f(z, s)$ in $V_{4 R, 4 R}$ we get

$$
\begin{aligned}
& \left|\left(\frac{\partial}{\partial z}\right) f(z, s)\right| \leqq \frac{1}{\delta} \sup _{z \in V_{R+\delta}}|f(z, s)| \quad \text { for } \quad(z, s) \in V_{R, R} \\
& \left|\left(\frac{\partial}{\partial z}\right)^{2} f(z, s)\right| \leqq \frac{2}{\delta^{2}} \sup _{z \in V_{R+\delta}}|f(z, s)| \quad \text { for } \quad(z, s) \in V_{R, R} .
\end{aligned}
$$

Then from (5.10), (5.14), (5.15) and this inequality, it is easily shown that the inequality

$$
\begin{aligned}
\left|\psi_{n}^{0}(z, s)\right| \leqq & \sum_{i=1}^{7} \sum_{i=n} \frac{n !}{n_{1} ! n_{2} ! n_{3} ! n_{4} ! n_{5} ! n_{6} ! n_{7} !} \frac{1}{\left(3 n-n_{1}-n_{2}\right) !}\left(c \rho|s|^{k+1}\right)^{n_{1}} \\
& \times\left({ }^{c}-\frac{c}{R} n_{2}|s|^{k+1}\right)^{n_{2}}\left(c^{2} \rho^{2}|s|^{2(k+1)}\right)^{n_{3}}\left(c \rho|s|^{k+1} \frac{c}{R} n_{4}|s|^{k+1}\right)^{n_{4}} \\
& \times\left(\frac{2 c^{2}}{R^{2}} n_{5}^{2}|s|^{2(k+1)}\right)^{n_{5}}\left(c \rho|s|^{k+1)}\right)^{n_{6}}\left(\frac{c}{R} n_{7}|s|^{k+1}\right)^{n_{7}}
\end{aligned}
$$

holds for $(z, s) \in V_{R, R}$. Since $j^{j} \leqq 3^{j} j$ !, for $\varepsilon>0$, the right hand side of (5.17) is less than

$$
\begin{gathered}
\sum_{\sum_{i=1}^{7} n_{i}=n} \frac{n !\left(2 n_{1}\right) !\left(2 n_{3}\right) !\left(2 n_{4}\right) !\left(2 n_{6}\right) ! n_{3} !(c / \varepsilon)^{2 n_{3}}}{n_{3} !} \frac{\left(c \rho|s|^{k+1}\right)^{n_{1}}}{\left(2 n_{1}\right) !} \\
n_{1} !\left(3 n-n_{1}-n_{2}\right) ! \\
\quad \times \frac{\left(\varepsilon \rho|s|^{k+1}\right)^{2 n_{3}}}{\left(2 n_{3}\right) !} \frac{\left(c \rho s^{k+1}\right)^{n_{4}}}{\left(2 n_{4}\right) !} \frac{\left(c \rho s^{k+1}\right)^{n_{6}}}{\left(2 n_{6}\right) !}
\end{gathered}
$$

Therefore we obtain for $(z, s) \in V_{R, R}$,

$$
\begin{aligned}
& \left|\psi_{n}^{0}(z, s)\right| \leqq C \sum_{j=0}^{n} \frac{n !(2 j) !}{j !(2 n) !} \frac{1}{2}(n-j+2)(n-j+1) \\
& \quad \times \sum_{n_{1}+n_{3}+n_{4}+n_{6}=j} \frac{\left(c \rho|s|^{k+1}\right)^{n_{1}}}{\left(2 n_{1}\right) !} \frac{\left(\varepsilon \rho|s|^{k+1}\right)^{2 n_{3}}}{\left(2 n_{3}\right) !} \frac{\left(c \rho|s|^{k+1}\right)^{n_{4}}}{\left(2 n_{4}\right) !} \frac{\left(c \rho|s|^{k+1}\right)^{n_{6}}}{\left(2 n_{6}\right) !}
\end{aligned}
$$




$$
\begin{aligned}
& \leqq C^{\prime} \sum_{j=1}^{n}\left(\frac{1}{2}\right)^{n-j} \frac{1}{(2 j) !}\left(\sqrt{c \rho|s|^{k+1}}+\varepsilon \rho|s|^{k+1}+\sqrt{c \rho|s|^{k+1}}+\sqrt{c \rho|s|^{k+1}}\right)^{2 j} \\
& \leqq C^{\prime \prime}\left\{\sum_{j=0}^{[n / 2]-1}\left(\frac{1}{2}\right)^{n-j} e^{4 \varepsilon \rho|s|^{k+1}}+\sum_{j=[n / 2]}^{n} \frac{1}{(2 j) !}\left(3 \sqrt{c \rho|s|^{k+1}}+\varepsilon \rho|s|^{k+1}\right)^{2 j}\right\} \\
& \leqq C^{\prime \prime \prime}\left\{\left(\frac{1}{2}\right)^{[n / 2]-2}+\frac{1}{(2[n / 2]) !}\left(3 \sqrt{c \rho|s|^{k+1}}+\varepsilon \rho|s|^{k+1}\right)^{2[n / 2]}\right\} e^{4 \varepsilon \rho|s|^{k+1}} .
\end{aligned}
$$

In the above inequality, we use the fact that for sosme constant $c^{\prime}$ the inequality $x<\varepsilon x^{2}+c^{\prime}$ holds for $x>0$.

From (5.18), $\varphi^{0}(z, s)=\sum_{n=0}^{+\infty} \psi_{n}^{0}(z, s)$ is a solution of the equation (5.9) $)_{0}$ with the initial condition (5.11), and we derive from (5.18): for $\varepsilon>0$,

$$
\left|\varphi^{0}(z, s)\right|<C e^{\varepsilon \rho|s|^{k+1}} \quad \text { for } \quad(z, s) \in V_{R, R} .
$$

Then by lemma 5.2 and (5.19), it is seen that for a sufficiently small $r_{0}>0$, the estimate

$$
\left|\left(\frac{\partial}{\partial s}\right)^{i}\left(\frac{\partial}{\partial z}\right)^{i \prime} \varphi^{0}(z, s)\right| \leqq C\left(\frac{1}{r_{0}}\right)^{i} \sup _{\left|s-s^{\prime}\right|<r_{0}} e^{\varepsilon \rho\left|s^{\prime}\right| k+1}
$$

hold for $(z, s) \in V_{R / 2, R / 2}$. Let $\varepsilon^{\prime}$ be a positive number such that $\varepsilon^{\prime}>\varepsilon$ and let $r_{0}=\rho^{-1 / k+1}$. Then there exist constant $C$ and $\rho_{1}$ such that

$$
\left|\left(\frac{\partial}{\partial s}\right)^{i}\left(\frac{\partial}{\partial z}\right)^{i^{\prime}} \varphi^{0}(z, s)\right| \leqq C \rho^{i /(k+1)} e^{\varepsilon \rho|s|^{k+1}}
$$

holds for $(z, s) \in V_{R / 2, R / 2}$ and $\rho \geqq \rho_{1}$.

For $\varphi^{j}(z, s)$, in the same way we obtain

$$
\left|\varphi^{j}(z, s)\right| \leqq C e^{\varepsilon \rho|s|^{k+1}} \sup _{\substack{|t| \leq \mid \mathrm{|l|} \\ z \in V_{4 R} j}} \rho^{-r(\lambda)+1}\left|G_{\rho}^{*}(z, t)\right| .
$$

Therefore by induction on $j$ from (5.3) it follows that the estimate (5.12) holds for $(z, s) \in V_{R_{j}, R_{j}}$ and $\rho \geqq \rho_{j+1}$.

Let $N$ be a large positive number such that

$$
N>M^{\prime}+2 M+4+3 \lambda .
$$

Then if we revert to variables $(y, s)$, proposition 5.1 implies that the estimate

$$
\left|\left(\frac{\partial}{\partial s}\right)^{i}\left(\frac{\partial}{\partial y}\right)^{i \prime} \varphi^{j}(y, s)\right| \leqq C \rho^{i / k+1} e^{\varepsilon \rho s^{k+1}}
$$

holds for $(y, s) \in V$ which is a sufficiently small neighvorhood of the origin in $\boldsymbol{R}^{2}$ and $\rho \geqq \rho_{0}$.

Not let us define $v$ and $f$ as follows:

$$
\left\{\begin{array}{l}
v(y, s) g(y, s) u_{\rho}^{N}(y, s) \\
f(z, s)=F(\rho y, \rho s),
\end{array}\right.
$$


where $g(y, s)$ is a smooth function defined in $\boldsymbol{R}^{2}$ with compact support contained in $V$ and equals to one in a subneighborhood of the origin, and $F(y, s)$ belongs to $C_{0}^{\infty}\left(\boldsymbol{R}^{2}\right)$ and satisfies $\iint e^{i y} F(y, s) d y d s=1$.

By (5.23) we get (shrinking $V$ if necessary); for some $\gamma>0$

$$
\begin{aligned}
P_{\rho}^{\prime} u_{\rho}^{N}= & 0\left(\rho^{-(N+1)}\right) \\
& \sum_{i+j \leq L}\left|\left(\frac{\partial}{\partial s}\right)^{i}\left(\frac{\partial}{\partial y}\right)^{j} u_{\rho}^{N}(y, s)\right| \leqq C \rho^{L} e^{-\rho\left(\gamma s^{k+1}+y^{2}\right)}
\end{aligned}
$$

holds for $(y, s) \in V$ and $\rho \geqq \rho_{0}$. Therefore by the standard method (c. f. see [6]) we know that the right hand side of (3.4) is less than $0\left(\rho^{-J^{\prime}}\right)$, where $J^{\prime}>2$ if we take $J$ large. On the other hand the left hand side of (3.4) is greater than $c^{-2}$, where $c$ is a non-zero positive constant. This is not compatible with lemma 3.2. Therefore ${ }^{t} P$ is not locally solvable at the origin This prove theorem 2.1-2.

\section{Proof of thorem 2.1-0.}

In this section we will prove the theorem 2.1-0. Our proof relies on the result of Grusin [5].

Let $P$ be an operator of (1.2) which satisfies the hypothesis of theorem 2.1-3, and let $P_{0}$ be an operator induced from $P$ :

$$
P_{0}\left(t, \frac{d}{d t}, \xi\right)=\left(\frac{d}{d t}+a t^{k} \xi\right)^{3}+\sum_{i+j \leq 2} B_{i, i}(0,0) t^{i+j(1+k)-3}\left(\frac{d}{d t}\right)^{i}(i \xi)^{j},
$$

where we denote $b_{i, j}(x, t) / t^{i+j(1+k)-3}$ by $B_{i, j}(x, t)$ which is a smooth function in a certain neighborhood of the origin by hypothesis.

Now we restate theorem 5.1 of [5] for the operator $P$.

Lemma 6.1. $P$ is hypoelliptic in some neighborhood of the origin if for all $\xi= \pm 1$. the equation $P_{0} u=0$ has no non-zero sulutiin in $\mathcal{S}(\boldsymbol{R})$. Here we denote by $\mathcal{S}(\boldsymbol{R})$ the space $\left\{f \in C^{\infty}(\boldsymbol{R}) ;{ }^{\forall} \alpha,{ }^{\forall} n,(1+|x|)^{n}\left(\frac{d}{d x}\right)^{\alpha} f(x) \rightarrow 0\right.$ as $\left.x \rightarrow \pm \infty\right\}$.

There is a following relation between local solvability and hypoellipticity.

Lemma 6.2. (see [11]) If the operator $* P$ is hypoelliptic in $V$, then $P$ is locally solvable at every point of $V$.

From the fact that if $P$ satisfies the condition of theorem $2.1-0$, then ${ }^{*} P$ also satisfies its condition and the above two lemmas, in order to prove theorem 2.1-0 it is sufficient to prove the following proposition.

Proposition 6.3. Let $Q$ be an operator of the form;

$$
Q\left(t, \frac{d}{d t}\right)=\left(\frac{d}{d t}-a t^{k}\right)^{3}+b_{1} t^{k-1} \frac{d}{d t} b_{2} t^{2 k-1}+b_{3} t^{k-2}
$$


where $a \in \boldsymbol{R}^{1}-\{0\}, b_{j} \in C^{1}$. Suppose that $k$ is even, then the equation $Q u=0$ has no non-zero solution in $\mathcal{S}(\boldsymbol{R})$.

We start from a lemma.

Lemma 6.4. Suppose that $k$ is even and $a$ is a non-zero real number. If $u, f \in \mathcal{S}(\boldsymbol{R})$ satisfy the equation $\left(\frac{d}{d t}-a t^{k}\right) u=f$, then $u$ can be written as follows

$$
u(t)= \begin{cases}\int_{-\infty}^{t} e^{a\left(t^{k+1}-s^{k+1}\right) /(k+1)} f(s) d s & \text { if } a<0 \\ \int_{+\infty}^{t} e^{a\left(t^{k+1-s k+1) /(k+1)}\right.} f(s) d s & \text { if } a>0\end{cases}
$$

Proof. $u$ can be written:

$$
u(t)=e^{a t^{k+1} /(k+1)}\left\{e^{-a t_{0}^{k+1} /(k+1} u\left(t_{0}\right)+\int_{t_{0}}^{t} e^{-a s^{k+1 /(k+1)}} f(s) d s\right\},
$$

where $t_{0}$ is an arbitrary real number. We assume that $a<0$. For $a>0$, we can prove it in the same way. Divide (6.3) by $e^{a t^{k+1 /(k+1)}}$, then we have

$$
e^{-a t^{k+1} /(k+1)} u(t)=e^{-a t_{0}^{k+1} /(k+1)} u\left(t_{0}\right)+\int_{t_{0}}^{t} e^{-a s^{k+1 /(k+1)}} f(s) d s .
$$

In this equation, let $t \rightarrow-\infty$, then the left hand side converges to 0 because $k$ is even. Therefore we get

$$
u\left(t_{0}\right)=-e^{a t_{0}^{k+1} /(k+1)} \int_{t_{0}}^{-\infty} e^{-a s^{k+1} /(k+1)} f(s) d s .
$$

Proof of proposition 6.3. We assume that $a<0$. For $a>0$, we can prove it in the same way. Let $u \in S(R)$ satisfy $Q u=0$. Then by lemma 6.4, we get

$$
u(t)=\int_{-\infty}^{t} \frac{1}{2}(t-s)^{2} e^{a\left(t^{k+1}-s^{k+1}\right) /(k+1)} f(s) d s,
$$

where $f(s)=-b_{1} s^{k-1} \frac{d u}{d t}-b_{2} s^{2 k-1} u-b_{3} s^{k-2} u$. (6.4) is integrated by parts to obtain

$$
\begin{aligned}
u(t)= & -\left(a b_{1}+b_{2}\right) \int_{-\infty}^{t} \frac{1}{2}(t-s)^{2} s^{2 k-1} e^{a\left(t^{k+1}-s^{k+1}\right) /(k+1)} u(s) d s \\
& -b_{1} \int_{-\infty}^{t}(t-s) s^{k-1} e^{a\left(t^{k+1}-s^{k+1}\right) /(k+1)} u(s) d s \\
& +\left\{(k-1) b_{1}-b_{3}\right\} \int_{-\infty}^{t} \frac{1}{2}(t-s)^{2} s^{k-2} e^{a\left(t^{k+1}-s^{k+1}\right) /(k+1)} u(s) d s .
\end{aligned}
$$

Let $t<0$, then

$$
\begin{aligned}
& \int_{-\infty}^{t} \frac{1}{2}(t-s)^{2} s^{2 k-1} e^{a\left(t^{k+1}-s^{k+1}\right) /(k+1)} d s \\
& =\left[\frac{1}{2}(t-s)^{2} s^{k-1}\left(-\frac{1}{a}\right) e^{a\left(t^{k+1}-s^{k+1}\right) /(k+1)}\right]_{-\infty}^{t}
\end{aligned}
$$




$$
\begin{aligned}
& +\frac{1}{a} \int_{-\infty}^{t}\left\{\frac{1}{2}(t-s)^{2}(k-1) s^{k-2}-(t-s) s^{k-1}\right\} e^{a\left(t^{k+1}-s^{k+1}\right) /(k+1)} d s \\
= & {\left[-\frac{1}{a^{2}}\left\{\frac{1}{2}(k-1)(t-s)^{2} s^{-2}-(t-s) s^{-1}\right\} e^{a\left(t^{k+1}-s^{k+1}\right) /(k+1)}\right]_{-\infty}^{t} } \\
& +\frac{1}{a^{2}} \int_{-\infty}^{t}\left\{s^{-1}-(k-2)(t-s) s^{-2}-(k-1)(t-s)^{2} s^{-3}\right\} e^{a\left(t^{k+1}-s^{k+1}\right) /(k+1)} d s \\
= & \frac{1}{a^{2}} \int_{-\infty}^{t}\left\{s^{-1}-(k-2)(t-s) s^{-2}-(k-1)(t-s)^{2} s^{-3}\right\} e^{a\left(t^{k+1}-s^{k+1}\right) /(k+1)} d s .
\end{aligned}
$$

Since $s<t<0$, we have $|s|>|t|$. Therefore we obtain

$$
0<-\int_{-\infty}^{t} \frac{1}{2}(t-s)^{2} s^{2 k-1} e^{a\left(t^{k+1}-s^{k+1}\right) /(k+1)} d s \leqq \frac{c_{1}}{|t|} \int_{-\infty}^{t} e^{a\left(t^{k+1}-s^{k+1}\right) /(k+1)} d s .
$$

In the same way, we get

$$
\begin{aligned}
& 0<-\int_{-\infty}^{t}(t-s) s^{k-1} e^{a\left(t^{k+1}-s^{k+1}\right) /(k+1)} d s \leqq \frac{c^{2}}{|t|} \int_{-\infty}^{t} e^{a\left(t^{k+1}-s^{k+1}\right) /(k+1)} d s \\
& 0<\int_{-\infty}^{t} \frac{1}{2}(t-s)^{2} s^{k-2} e^{a\left(t^{k+1}-s^{k+1}\right) /(k+1)} d s \leqq \frac{c_{3}}{|t|} \int_{-\infty}^{t} e^{a\left(t^{k+1}-s^{k+1}\right) /(k+1)} d s .
\end{aligned}
$$

Since $k$ is even and $a<0$, from (6.5)-(6.8) it follows that there exists a positive number $T>0$ such that the inequality

$$
\sup _{t \in(-\infty,-T]}|u(t)| \leqq \frac{1}{2} \sup _{t \in(-\infty,-T]}|u(t)|
$$

holds. From this inequality, we get

$$
\sup _{t \in\left(-\infty,-T_{?}\right.}|u(t)|=0 \text {. }
$$

Therefore by the uniqueness of solution of ordinary differential equation, we obtain $u(t)=0$ for all $t \in \boldsymbol{R}^{1}$.

\section{Acknowledgement.}

I wish to express my gratitude to Professor S. Mizohata for his valuable suggestions.

\section{DePaRTMENT OF MATHEMATICS KYOTO UNIVERSITY}

\section{References}

[1] R. Beals \& Fefferman: On local solvability of linear partial differential equations. Ann. Math. 97, 482-498 (1973).

[2] F. Cardoso \& F. Treves: A necessary condition of local solvability for pseudodifferential equations with double characteristics: Ann. Inst. Fourier, 24-1, 225-292 (1974). 
[3] Ju. V. Egorov: On solvability condition for differential equations with simple characteristics: Soviet math. dokl. 17, 1194-1197 (1976).

[4] R. Goldman: A necessary condition for the local solvability of a pseudo-differential equation having multiple characteristics. J. Diff. Eq. 19, 176-200 (1975).

[5] V. Grusin: On a class of hypoelliptic operators: Math. USSR. sb. 12, 458-476 (1970).

[6] L. Hormander: Linear partial differential operators. (1963).

[7] V. Ja. Ivrii: Differential equations with multiple characteristics and with no solutions: Soviet math. Dokl. 12, 769-772 (1971).

[8] S. Mizohata: Solutions nulles et solutions non analytiques. J. Math. Kyoto Univ. 1, 271-302, (1962)

[9] L. Nirenberg \& F. Treves: On local solvability of linear partial differential equations. I. Necessary condition, II Sufficient condition Comm. pure and appl. Math. 23, 1-38, 459-509 (1970). Correction: Ibid. 24, 279-288 (2971).

[10] F. Treves: On the local solvability of linear partial differential equations : Russian Math. Survey, 29-2, 263-292 (1974).

[11] F. Treves: Topological vector spaces, Distributions and Kernels: (1967). 